\title{
Models of information behavior: changes in psychological boundaries of internet users
}

\author{
Irina Abakumova ${ }^{1}$, Anastasia Grishina ${ }^{1, *}$, Galina Zvezdina $^{1}$, Ekaterina Zvezdina ${ }^{1}$, Elena \\ Dyakova $^{2}$ \\ ${ }^{1}$ Don State Technical University, Gagarina sq, 1, Rostov-on-Don, Russia \\ ${ }^{2}$ Rostov State Transport University, Rostovskogo Strelkovogo Polka Narodnogo Opolcheniya Sq., 2, \\ Rostov-on-Don, Russia
}

\begin{abstract}
The authors conducted a study aimed at studying the characteristics of students with different models of information behavior in connection with the changes in psychological boundaries when using Internet technologies. The article reveals the role of information technologies and technical means, which become an important component of the life of a modern person and change his living space. The authors show the influence of information technologies on mental processes and relationships of people and consider the features of information behavior in connection with possible violation of psychological boundaries when using technical means The article analyses the problem of diagnosing information behavior and contains an overview of diagnostic methods of studying Internet addiction. It's shown that in modern world information behavior is needed to be studied outside the context of addictive behavior. In the empirical part of the study authors distinguish the models of information behavior: entertaining, informational, communicative, productive and pragmatic. The empirical study defines the main behavioral characteristics of the presented information behavior models and investigate the changes in psychological boundaries when using the Internet among students with different models of information behavior. The results of the study allow to reveal the subjective attitude of students to the possibilities and availability of Internet resources in connection with differences in the severity of the expansion of psychological boundaries in communication, in the reflection of violation of boundaries, in changing needs.
\end{abstract}

\section{Introduction}

The involvement of a modern person to the information space changes his attitudes, behavior, lifestyle. The information society sets certain guidelines for social relations and contributes to the emergence of human information behavior.

In the scientific community, there are discussions about the legality of using the term "information behavior". However, discussions on this issue in foreign psychology led to the popularization and even wider use of this concept.

\footnotetext{
* Corresponding author: avgrishina.donstu@gmail.com
} 
Modern russian researchers are actively studying information behavior and its various aspects. The information behavior of readers and their information needs were actively studied in the $60-90$ s by Russian librarians and bibliologists (Yu.A. Shreider, E.S.Bernshtein, E.L.Shapiro, T.N. Koltypina). The pedagogical aspect of the information behavior of specialists was studied by E. Kulakova. The culture of information behavior of Internet users from a philosophical and cultural approach was considered by Matvienko D.V., the culture of information behavior as a structural part of information culture is reflected in the works of Antonova G., Vokhrysheva M.G., Gorlova I.I., Degterev A.R., Dulatova A.N., Zinovieva N.B., Zubova Yu.S., Kudrina T.A., Lotman Yu.M., Petrova V.M. [Dresher Y. connects information behavior with the efforts and actions that a person makes to obtain and master information and create new knowledge (Y. Dresher) [1, 2,3].

Information behavior is manifested in the ability to navigate in the information space, in the skills of mastering information technologies, in the ability to adequately assess and productively use the information received. The ability to create, distribute and be responsible for the dissemination of new information products also lies within the competence of information behavior. Although the technical side of informational behavior is significant, this phenomenon cannot be considered one-sided, psychological aspects are an important component. The lack of research on this aspect of information behavior actualizes the need to study and disclose the psychological characteristics of information behavior and its relationship with the peculiarities of using technical means and the Internet.

The World Wide Web is considered today not only as an information infrastructure, but as a new virtual space, where it is possible to fulfill the urgent needs of both individuals and large groups. The openness of the Internet provides great opportunities for a person to realize his needs and motives, and the interactivity of this information tool allows you to "touch" various events in real time.

On the Internet a person reveals himself through his informational behavior, on the one hand, as a consumer, and on the other, as a creator and distributor of information. At the same time, the information behavior reflects the degree of accessibility and comfort of using information resources [4].

However, accessibility and comfort are often factors of uncontrolled use of technical means and violation of the psychological boundaries between a person and technical means.

The active use of technical means and information and communication technologies leads to the blurring of psychological boundaries between a person and technical devices, contributes to the formation of psychological and technological dependence. A person begins to perceive technical devices as a necessary part of life, and even as a part of himself. From tool functions, a technical device turns into a vital function, without which the usual way of life is disturbed. A person experiences discomfort in the absence of the opportunity to use technical devices.

The introduction of technical devices into the life of a modern person has an impact on the motivational-needs sphere, "new technical means form new needs, and also change the ways of meeting existing needs. In addition to the main function, technical means begin to perform other functions - to indicate the status of a person, the degree of his acquaintance with technology" [5].

Technical devices mediate different types of activities - professional, educational activities, communication, play. The active use of these means changes a person's attitude to these means, they begin to be perceived as a part of a person without which he cannot imagine his life, and their multifunctionality requires a constant increase in digital competence and the development of new skills and abilities [6].

A change in psychological boundaries among active users of information and communication technologies entails a change in the space of life. According to A. Kotlyarov, virtual reality is a special, "intermediate" psychological "space". It reflects the specific 
perception of the computer and can be different, depending on the needs of the person. Due to virtual reality, a person continues his inner reality in the direction that corresponds to his goals. Virtual reality fills "empty" places in a person's life, creates an opportunity to "change, improve and create" in place of ordinary reality a new one, designed to order. The "new" reality makes it possible to achieve one's goals in it and is therefore attractive. Thus, virtual reality is not created in order to completely repeat ordinary life, its task is to fill the "gaps in the gaps" of reality, ordinary life, to "repair" the external and internal world in accordance with its goals [7].

Involvement in traveling on the Internet from one site to another leads to the fact that people begin to perceive computers as an extension of their personality in this "space", reflecting their tastes and interests. Such a state can be so overwhelming that at times there is a dissolution of their own "Self" . This opens the door to the various fantasies and transference reactions that can be projected onto this space [8].

In connection with the widespread prevalence of information and communication technologies, and in particular the Internet, it becomes urgent to study the features of information behavior of active Internet users.

\section{Research methodology and methods}

The study of information behavior within the framework of psychology began as early as the 70 s and 80 s of the twentieth century: the emphasis in research gradually shifted from studying communication mediated by computers to analyzing the characteristics of user activity on the Internet and building models of Internet behavior.

Diagnostic approaches to this problem have their own characteristic features. The first proposed diagnostic methods considered behavior on the Internet in the context of the Internet addiction formation. Thus, K. Young, having proposed his first version of the Internet Addiction Test (IAT) in 1994, uses the concept of "Internet addiction" to indicate the destructive effect of information technology on a person, comparing this addiction with an obsessive state. This technique does not deliberately differentiate between content that leads to obsessive behavior. The test can be used as a rapid diagnostic method to determine the presence / absence of addiction (Young, 1998).

Further study of Internet addiction and its specifics leads K. Young to the conclusion that Internet addiction can be caused by different reasons and manifest itself in different ways, requiring different types of treatment. The author supplements her IAT methodology (up to 20 questions) by introducing assessments related to the following areas: avoidance of reality, obsession, neglection of duties, anticipation, lack of control, social avoidance.

The diagnostic toolkit, developed in the late 90s of the XX and in the early 2000s, focuses on the study of dependent information behavior, and the presented methods are modifications of the scales of K. Young and S. Chen.

Since the 2000s, the availability of the Internet began to grow, thanks to the revolution in the development of technical means (the emergence of smartphones and the mobile Internet). The emphasis in the diagnosis of information behavior is gradually shifting from determining the presence of dependence on the Internet, to the study of online behavior and its influence on the personal characteristics of the user, the model of his information behavior.

The proposed study focuses on the study of information behavior models. As a research methodology, the author's questionnaire was used, which is based on the idea of studying the motivational orientation of Internet users.

To study psychological boundaries when using the Internet, we used a method to assess changes in psychological boundaries when using technical means (MIG-2 TS) (Rasskazova, A.Sh. Tkhostov), 
In order to study the tolerance to uncertainty in students with different models of information behavior, the McLane's Tolerance to Uncertainty Scale methodology was applied in the adaptation of E.G. Lukovitskaya. According to E. Frenkel-Brunswick, tolerance to uncertainty is manifested in such individual characteristics as cognitive style, belief system, interpersonal communication and social interaction, behavior when solving problems.

\section{Results}

As part of the study of information behavior, an empirical study was carried out in order to identify the features of information behavior of students with different motivational orientation in connection with the change in psychological boundaries when they use Internet technologies. Psychology students in the amount of 105 people were the respondents of the study. The average age of the subjects was 22 years. Boys and girls who study at daytime and evening education took part, of whom girls made up more than $80 \%$ of the respondents.

The research was carried out using the following methods: observation, interview, psychological diagnostics. The following methods were used: questionnaire, "Methodology for assessing changes in psychological boundaries when using technical means" (MIG-2 TS) (E.I. Rasskazova, V.A.Emelin, A.Sh. Tkhostov), "Scale of tolerance to Uncertainty"McLane (MSTAT-I) as adapted by EG. Lukovitskaya. The diagnostic results were processed using the methods of mathematical statistics.

Analysis of the results on the questionnaire for studying the use of resources of the Internet by students showed the following results. When studying the target orientation of the students Internet use, the following areas can be distinguished: a third of the respondents $(29.9 \%)$ define what is important for themselves as finding the necessary information (viewing and reading news), about a third (28.9\%) of the respondents use the Internet resource for communication on social networks, more than $20 \%$ of students use for entertainment and $10.3 \%$ of students use for educational purposes. The remaining $10 \%$ was distributed approximately evenly for the following purposes - for buying / selling goods, in order to share information, and more.

According to the results of empirical research, respondents spend most of their time on social networking (35.1\%), reading and viewing news is preferred by $22 \%$ of respondents, more than $20 \%$ are looking for information for their needs and $14.4 \%$ of students are actively studying in online format, participate in marathons, webinars.

Thus, the dominant motives of students going online are motives of communication, cognitive and entertainment motives. The results of the study showed that only one tenth of students use the Internet for teaching purposes. About $62 \%$ of students noted that they use the Internet for teaching purposes when necessary, and $35 \%$ indicated that they turn to Internet resources on a daily basis when teaching.

In our opinion, it is the motivation of users that is the most important link in behavioral models on the Internet. Understanding motivation as the determination of behavior as a whole, it is necessary to include in the concept of motivation all types of motives - motives, needs, interests, aspirations, goals, drives, attitudes and others [9]. This understanding of motivation as a complex of factors influencing and determining behavior has led us to identify five models of Internet behavior: entertainment, informational, communicative, productive, pragmatic. It should be emphasized that within the framework of the author's motivational approach to models of Internet behavior, the content consumed itself is not so important as the motive for using this content.

Each model is distinguished by a certain direction of behavior and a complex of different motives of behavior. 
Behavior within the entertainment model is aimed at the process of activity and at oneself: the user himself receives knowledge, emotions, impressions in the process of consuming entertainment content. This model includes motives associated with the use of information to maintain or change their emotional state, as well as in order to just pass the time and be distracted.

Within the framework of the informational model, the user's behavior is aimed at the result of the activity and at oneself. This model is the classic consumption of information from the Internet to solve their educational, professional and even everyday tasks. In this case, the emphasis is on the search result, and not on its process, as in the entertainment model.

The communicative model of Internet behavior includes all forms of behavior that involve interaction with other users (both active and passive). In this case, the activity is directed at oneself and at others, and the process of communication is more important than its result.

A productive model presupposes the creation of new content, a product of one's own creativity, which is important to share with others, i.e. in this case, the activity is aimed at results for others.

Within the framework of the pragmatic model of Internet behavior, 2 types of behavior can be distinguished: 1) users who sell something and thus earn; 2) users who buy something, thereby meeting their needs.

Based on the presented theoretical model, the author's methodology for studying behavior patterns on the Internet was developed. The technique has passed an expert assessment and is being tested [10].

Based on the results of studying the motivational orientation of information behavior, the respondents were divided into groups: entertainment, informational, communicative, productive, pragmatic.

In selected groups with different motivational orientation of information behavior, the severity of tolerance to uncertainty was studied. According to the diagnostic results, the group with a productive model of informational behavior showed the highest level of tolerance to uncertainty. The ability to withstand a state of uncertainty without dysfunctional heightening of anxiety was demonstrated by students with a constructive creative focus.

\section{Tolerance to uncertainty}

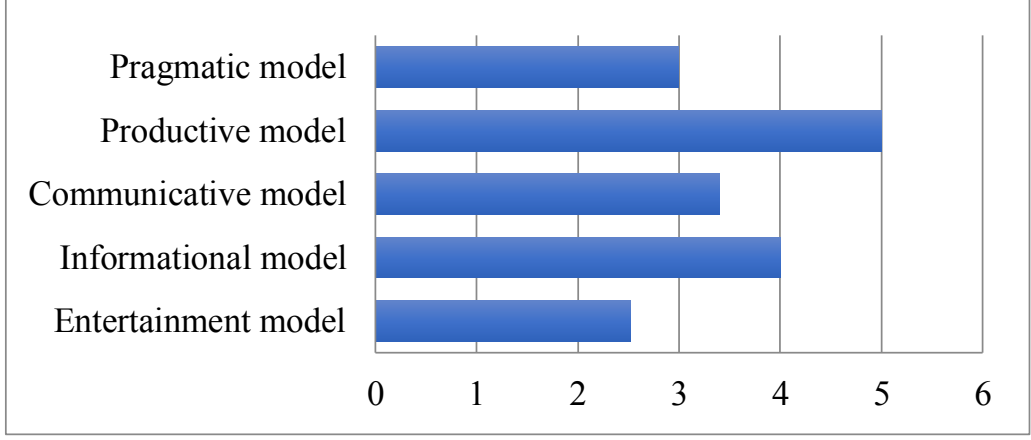

Fig. 1. The severity of tolerance to uncertainty among respondents from groups with different models of information behavior.

The informational model showed average results on the uncertainty tolerance scale. The rest of the groups of subjects showed below average and low points on the uncertainty tolerance scale. Such respondents feel uncomfortable in difficult, ambiguous, uncertain 
situations, are afraid of the unknown, try to bring maximum clarity and unambiguity into their lives and the environment, often by artificially simplifying complex reality (Osin, 2010).

Using the methodology for assessing the change in psychological boundaries when using technical means (MIG2-TS) (E.I. Rasskazova, V.A.Emelin, A.Sh. Tkhostov), we studied the subjective attitude of the selected groups of students to the possibilities and availability of resources the Internet. The indicator "Expanding the boundaries in communication" for all groups of students is within the average values. A large degree of this scale is observed in two groups of students with entertaining and pragmatic leading motivation,. These results indicate that the respondents of these groups are more inclined to surf in the Internet for a long time. It is important for them that the ability to access the Internet was always uninterrupted and constant.

The smallest expansion of psychological boundaries is observed in the group with informational motivation of behavior, which may indicate that Internet technologies are used as a tool and information consumption is controlled by respondents.

The subjective feeling of the accessibility and attainability of others using the Internet does not have pronounced differences among groups of students with different motivational orientation of information behavior, as evidenced by the coefficient U- the Mann-Whitney criterion. Statistical verification of the significance of differences in the results obtained in the studied groups showed that with a high degree of probability it can be asserted $(p=0.05)$ that significant differences in the severity of changes in psychological boundaries when using Internet technologies in groups with different information behavior models were not revealed.

The indicator "Reflection of violation of boundaries" received higher scores in groups with entertaining and informational models of behavior. For groups with communicative and productive information behavior, this scale is within the average values. A low level of reflection of violation of psychological boundaries is expressed in the pragmatic model. Getting positive emotions from shopping on the Internet possibly weakens the control and analysis of one's own behavior.

According to the results obtained, respondents with entertainment and informational models of Internet behavior more often reflect and control the negative effects of using Internet technologies than respondents with pragmatic motivation. Reflection of boundary violations can act as a buffer, weakening the connection between boundary changes and adaptation violations.

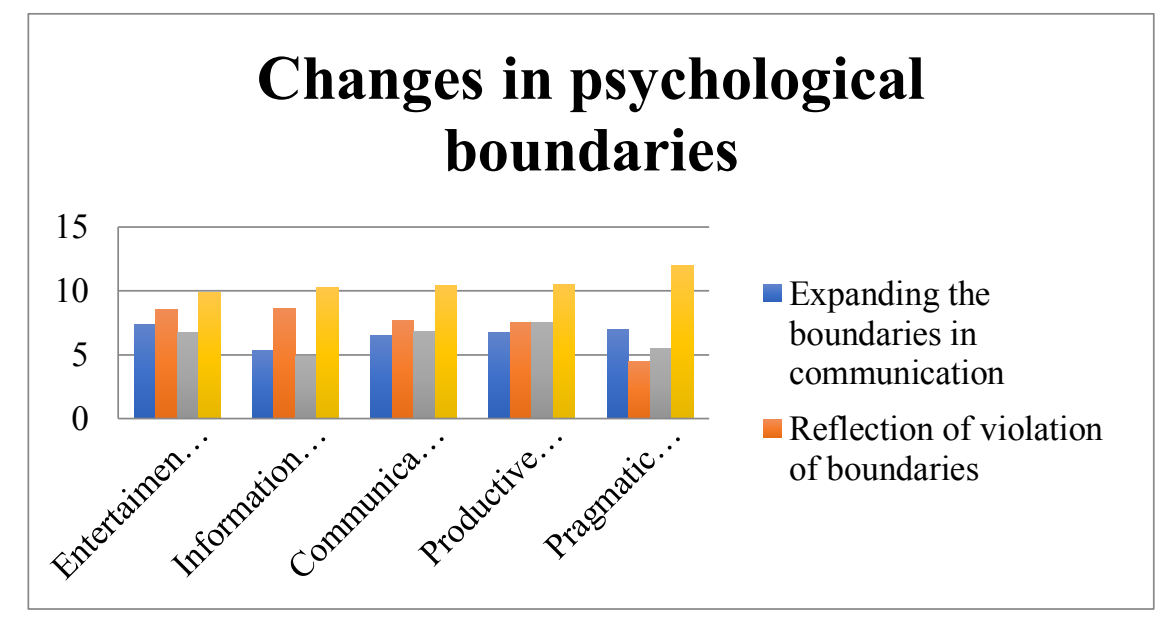

Fig. 2. The severity of changes in psychological boundaries when using the Internet among respondents with different models of information behavior. 
The study of the preferences of technology - simplicity and possibilities showed that the severity of these indicators is within the average values for all groups of respondents. The preference for simplicity technology is least expressed among students with informational motivation for Internet behavior.

Students with the dominance of the informational model of behavior are characterized by a less pronounced expansion of psychological boundaries and a more pronounced reflection of behavior. Among the representatives of this group, such indicators as "preference for technology-simplicity" and "preference for technology-opportunities" are least expressed. This model is characterized by the consumption of information from the Internet to solve their educational, professional and even everyday tasks. At the same time, the emphasis is on the result of the search, and not on its process, as in the entertainment model, which is probably why they have a higher reflection of violation of boundaries. The focus on the result acts as a kind of regulatory mechanism in the search activity of this group of respondents. We can say that this group of subjects is more focused on technology and the network - the Internet as a tool and resource. The analysis of the possibilities of the Internet environment is carried out more by representatives of a pragmatic orientation. In the remaining groups of subjects, there are no obvious differences in the severity of these indicators.

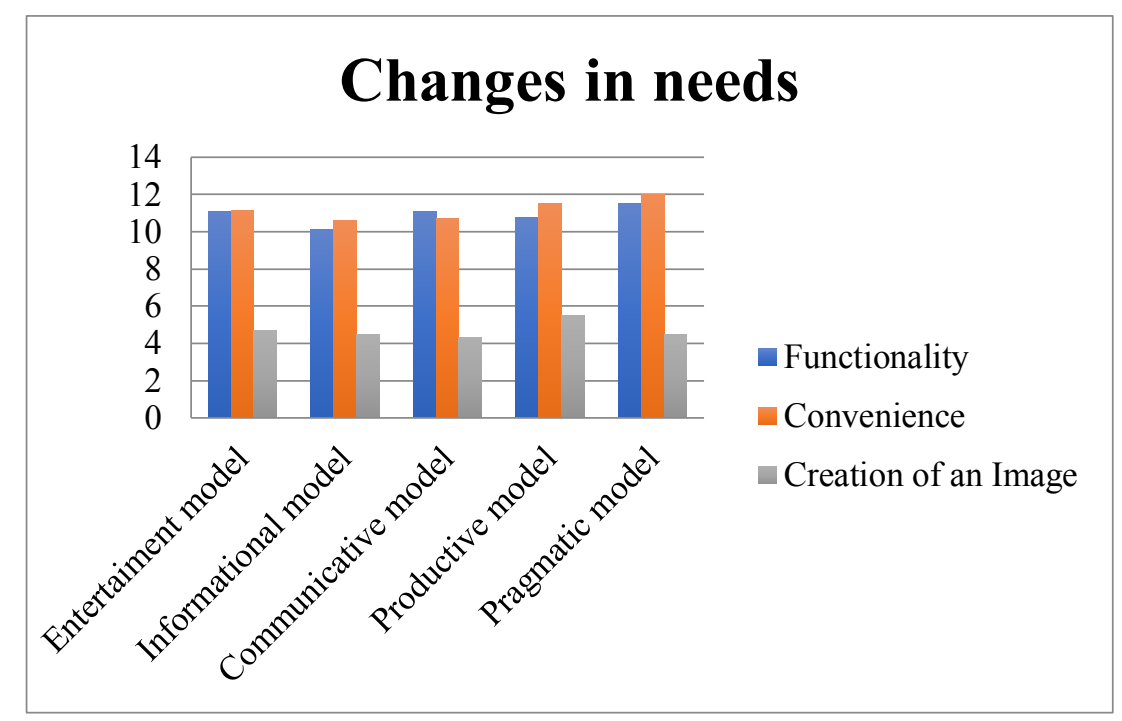

Fig. 3. The severity of changes in needs when using the Internet among groups with different models of information behavior.

The study of the subjective feeling of changes in the needs of students in connection with the active consumption of the Internet showed that the functionality of the Internet in the eyes of the respondents is increasing. It becomes subjectively obvious to the user that the ability to contact any person at any time is vitally necessary, that it is almost impossible to do without a quick and convenient search for information. Functionality it is especially important for a pragmatic model of information behavior, according to the results obtained. The subjective value of the characteristics of technical means associated with simplicity, convenience, lack of unnecessary details, practicality is important for all models of information behavior, most of all it is relevant for a pragmatic model of information behavior. The functionality and convenience of the Internet environment of the sites visited are important when searching / buying / selling goods and services.

The scale "Creation of an Image" on the Internet among the studied groups of students received the least points in comparison with the previous scales. Students of a productive 
model of behavior are more concerned with reflecting their image on the Internet than other groups. This fact can be explained by the desire of the respondents of the productive model to present themselves and their product in a more favorable way.

The study of Spearman's correlations between the models of information behavior and the scale indicators of the change in psychological boundaries when using Internet technologies made it possible to identify the links between these parameters. A positive correlation was revealed between the indicators of the pragmatic model of information behavior and the subjective value of functionality and convenience $(r=0.47$, at $\mathrm{p}<0.05)$ and the preference for technology-opportunity $(\mathrm{r}=0.53$, at $\mathrm{p}<0.05)$. No other significant relationships were found between the studied parameters.

\section{Discussion}

One of the main goals of this study was to find the connection between a leading motive for a user to go online and psychological changes a person might have.

The achieved results fully support our hypothesis, as the correlation between motives and psychological changes was found and statistically proved. Though not all of the results appeared profound the overall direction of results showed trends that could be helpful to build multicomponent models of internet behavior, that is an important part of our new reality.

Previous studies support the data, presented in Results: many authors reveal negative psychological consequences of using the Internet. E.Aboujaoude shows that impulsivity, narcissism, and aggression are some of the personality traits that seem to be nurtured by the Internet, with possible negative offline consequences [11].

Some authors focus on negative psychological traits that manifest themselves brighter online than in real life. These facts can be also considered as psychological consequences of using the Internet, closely connected with the motives of behavior. A.Kurek, P.E.Jose, J.Stuart have shown that three dark personality traits (narcissism, psychopathy, and sadism), as well as false self, were positively associated with online disinhibition. Perceptions of false self have been found to be a significant predictor of cyber aggression when mediated by online disinhibition. In the case of cyber aggression, however, psychopathy, sadistic traits, and online disinhibition were found to be significant predictors of this outcome [12]. T.C.Marshall, K.Lefringhausen, N.Ferenzi revealed that extraverts more frequently updated about their social activities and everyday life, which was motivated by their use of Facebook to communicate and connect with others. People high in openness were more likely to update about intellectual topics, consistent with their use of Facebook for sharing information. Participants who were low in self-esteem were more likely to update about romantic partners, whereas those who were high in conscientiousness were more likely to update about their children [13].

Expansion and "blurring" of psychological boundaries was explored in the context of self-disclosure in the Internet and especially in social networks. N.N.Bazarova, Y.H.Choi introduces the functional model of self-disclosure on social network sites: people pursue strategic goals and disclose differently depending on social media affordances, and selfdisclosure goals mediate between media affordances and disclosure intimacy [14]. S.Trepte, L.Reinecke proposed that the disposition for online self-disclosure had a positive longitudinal effect on social network sites use which in turn positively influenced the disposition for online self-disclosure [15].

\section{Conclusion}

Based on the conducted empirical research, the following conclusions can be drawn: 
- it was revealed that the dominant motives for going online for students are motives of communication, cognitive and entertainment motives;

- it was found that tolerance to uncertainty is more pronounced among students with productive and informational models of behavior;

- it was found that students with different models of information behavior clearly differ in the severity of violations of psychological boundaries when using the Internet, but these differences are not statistically significant;

- it was found that the greatest loss of privacy when using the Internet is observed in a group with an entertaining motivation for informational behavior:

- changes in the area of needs among respondents of all groups are characterized by an increase in the subjective significance of additional functions: the perception of a technical device as part of the image, the requirement for its complexity or convenience, multifunctionality becomes important.

The study made it possible to reveal the features of the information behavior models in connection with the change in the psychological boundaries of respondents in using the Internet and outline the prospects for further study of this problem.

\section{Limitations}

Our research has some limitations. We didn't take into account the age and gender characteristics of the respondents, but obviously, informational consumption among representatives of different generations may vary due to different reasons.

\section{Acknowledgements}

The research was supported by the Russian Foundation for Fundamental Research (Project No. 18-29-22004) (2018) «Psychological and genetic studies of users' behavior predictors that determine the perception of Internet content of various informational orientation».

\section{References}

1. E.V. Kulakova, Information behavior of specialists: essence and ways of studying. Abstract of Cand. dis. in ped. sciences (SPbGUKI, SPb, 2000) https://www.km.ru/referats/2B4568E1B3F04A5D82952ED4DE5F204A

2. D.V. Matvienko, The culture of information behavior of Internet users: a philosophical and cultural research: Abstract of Cand. dis. in philos. sciences (Krasnodar, 2009)

3. Yu.N. Dresher, T.A. Atlanova, Scientific and technical libraries 11, 5-14 (2005) http://ellib.gpntb.ru/subscribe/index.php?journal

4. Yu. Potekhina, L. Belyakova, Bulletin of the Khabarovsk State Academy of Economics and Law 1, 115-119 (2001)

5. E.I. Rasskazova, V.A. Emelin, A.Sh. Tkhostov, Psychological model of the consequences of the information technology use. Diagnostics of the psychological consequences of the influence of information technology on a person (Akropol, Moscow, 2015)

6. V.A. Emelin, E.I. Rasskazova, A.Sh. Tkhostov, National Psychological Journal 1(7), 8187 (2012)

7. A.V. Kotlyarov, Other Drugs, or Homo Addictus: The Addicted Man (Psychotherapy, Moscow, 2006) 
8. E.N. Kryukova, The journal Bulletin of the Samara State Technical University. Series: Psychological and pedagogical sciences 1(17), 87-92 (2012)

9. N.I. Meshkov, Integration of education 9(1), 37-43 (2015) doi: 10.15507/Inted.078.019.201501.037

10. I.V. Abakumova, A.V. Grishina, G.P. Zvezdina, SHS Web of Conferences 70, 01002 (2019) https://doi.org/10.1051/shsconf/20197001002

11. E. Aboujaoude, Journal of Behavioral Addictions 6(1), 1-4 (2017) https://doi.org/10.1556/2006.6.2017.009

12. A. Kurek, P.E. Jose, J. Stuart, Computers in Human Behavior 98, 31-40 (2019) https://doi.org/10.1016/j.chb.2019.03.027

13. T.C. Marshall, K. Lefringhausen, N. Ferenzi, Personality and Individual Differences 85, 35-40 (2015) https://doi.org/10.1016/j.paid.2015.04.039

14. N.N. Bazarova, Y.H. Choi, Journal of Communication 64(4), 635-657 (2014) https://doi.org/10.1111/jcom.12106

15. S. Trepte, L. Reinecke, Computers in Human Behavior 29(3), 1102-1112 (2013) https://doi.org/10.1016/j.chb.2012.10.002 